\title{
Nanoparticle Surface Characteristic
}

National Cancer Institute

\section{Source}

National Cancer Institute. Nanoparticle Surface Characteristic. NCI Thesaurus. Code C62349.

A distinguishing feature present on the surface of a nanoparticle. 